\title{
Aneurisma de arteria testicular: reporte de un caso y revisión de la literatura
}

\author{
Edgar O. Durán-Sánchez¹, César A. Mendivil-Guerrero², Juan C. García-Norzagaray, \\ Jesús A. Villalobos-López ${ }^{1}$ y Jaime G. Valle-Leal* \\ ${ }^{1}$ Departamento de Cirugía General, Hospital General Regional Número Uno, Instituto Mexicano del Seguro Social; ${ }^{2}$ Departamento de Angiología \\ y Cirugía Vascular, Unidad Médica de Alta Especialidad 167; ${ }^{3}$ Departamento de Enseñanza e Investigación, Hospital General Regional Número \\ Uno, Instituto Mexicano del Seguro Social. Ciudad Obregón, Sonora, México
}

\begin{abstract}
Resumen
Introducción: El aneurisma de la arteria testicular es poco frecuente; el término se describe como la dilatación de cualquier vaso sanguíneo en el cuerpo. Caso clínico: Se presenta el caso de un paciente de 15 años con un aneurisma de la arteria testicular izquierda sin ningún antecedente de importancia familiar ni personal, quien se presentó a consulta por aumento de volumen en la región inguinal de larga evolución, acompañado de dolor esporádico y sin más síntomas. Se diagnosticó hernia inguinal y se procedió a cirugía. El diagnóstico se realizó de manera posoperatoria por medio de estudio histopatológico. En este caso, se destaca la presentación de un aneurisma verdadero de la arteria testicular y el resultado después del tratamiento quirúrgico definitivo. Conclusiones: La etiología del aneurisma y del pseudoaneurisma reportada en la literatura se describe posterior a un traumatismo testicular, y en pocos casos de origen congénito. Las manifestaciones clínicas pueden ser dolor y una masa inguinal, y muchas de las veces pueden confundirse con hernias inguinales o ser una patología agregada, por lo que el abordaje de los pacientes con patología inguinal o testicular debe ser protocolizado e incluir el aneurisma dentro de los diagnósticos diferenciales.
\end{abstract}

Palabras clave: Aneurisma testicular. Arteria testicular. Masa inguinal.

\section{Testicular artery aneurysm: case report and review of the literature}

\section{Abstract}

Background: Aneurysm of the testicular artery is a rare entity; the term is described as the dilation of any blood vessel in the body. Case report: An 18-year-old patient with a left testicular artery aneurysm, with no family or personal history of medical importance, presented for consultation due to a volume increase of long evolution in the inguinal region, accompanied by sporadic pain with no other symptoms. The testicular artery aneurysm was not detectable preoperatively by ultrasound, which only reported data compatible with left inguinal hernia and varicocele. The diagnosis was made postoperatively by a histopathological study. This case highlights the presentation of a true aneurysm of the testicular artery and the result after definitive surgical treatment. Conclusions: The etiology of the aneurysm and pseudoaneurysm reported in the literature is described after a testicular trauma and, a few cases of congenital origin. The clinical manifestations are pain and an inguinal

\section{Correspondencia:}

*Jaime Guadalupe Valle-Leal

E-mail: valle_jaime1@ @otmail.com
Fecha de recepción: 15-03-2018

Fecha de aceptación: 21-06-2018

DOI: 10.24875/BMHIM.18000017
Disponible en internet: 01-11-2018 Bol Med Hosp Infant Mex. 2018;75:373-376

www.bmhim.com

1665-1146/@ 2018. Hospital Infantil de México Federico Gómez, impreso por Permanyer México SA de CV, todos los derechos reservados. 
mass, which can be frequently confused with inguinal hernias or an aggregated pathology. Therefore, the approach of patients with inguinal or testicular pathology should be protocolized and include aneurysm within the differential diagnoses.

Key words: Aneurysm. Testicular artery. Inguinal mass.

\section{Introducción}

El término "aneurisma» describe la dilatación de cualquier vaso sanguíneo. La incidencia de este padecimiento aumenta con la edad y, por lo general, es resultado de enfermedades degenerativas, procesos inflamatorios, infecciosos, genéticos o traumáticos ${ }^{1-3}$.

El aneurisma de la arteria testicular es muy poco frecuente. Existen muy pocos reportes en el mundo, por lo que se carece de información al respecto. Sobre los reportes encontrados, en la mayoría de los casos se trata de pseudoaneurismas secundarios a un traumatismo testicular. El diagnóstico se integra de forma clínica y por estudios de imagen: generalmente ultrasonografía Doppler, tomografía computarizada o resonancia magnética ${ }^{1-4}$.

Básicamente, tres arterias realizan la irrigación de cada testículo: la arteria testicular, la arteria del conducto deferente y la arteria cremastérica. La arteria testicular sale directamente de la aorta abdominal, justo por debajo de la arteria renal. Como todas las estructuras que van y vienen de los testículos, conforma el cordón espermático que se inicia en el anillo inguinal profundo y termina en el borde posterior del testículo. La arteria del conducto deferente se origina de la arteria vesical inferior y acompaña de cerca al conducto deferente, que también hace parte del cordón espermático. La arteria cremastérica se origina de la arteria epigástrica inferior e irriga el músculo cremáster $^{5}$. La arteria testicular es la principal fuente de aporte sanguíneo al testículo. Existen anastomosis entre los vasos testiculares y deferentes, y también se dan algunas anastomosis entre estas y las arterias cremastéricas en aproximadamente dos tercios de los pacientes. Parecen existir anastomosis adicionales entre los vasos testiculares, cremastéricos y escrotales ${ }^{6}$.

La descripción de este caso tiene como objetivo que el médico clínico y el radiólogo puedan considerar al aneurisma de la arteria testicular dentro de los diagnósticos diferenciales de pacientes de todas las edades con orquialgia, sobre todo en aquellos casos en los que exista el antecedente de traumatismo de la región testicular.

\section{Caso clínico}

Paciente de sexo masculino, de 15 años de edad, sin ningún antecedente familiar ni personal de importancia; desarrollo sexual en estadio III de Tanner.

Inició su padecimiento actual un mes previo a su cirugía, con dolor y aumento de volumen en la región inguinal izquierda. Se le realizó ultrasonido inguinoescrotal que reportó hernia inguinoescrotal izquierda no estrangulada, vascularidad testicular bilateral con características normales y datos ecográficos sugestivos de varicocele izquierdo, motivo por el que se decidió programar para cirugía.

Ante la exploración inguinal izquierda, los hallazgos fueron hernia inguinoescrotal izquierda con saco herniario que contiene epiplón de $15 \mathrm{~cm}$ y anillo de $3 \mathrm{~cm}$ que acompaña a cordón espermático, el cual se cortó y ligó en su base; venas del plexo pampiniforme con dilatación importante, de $5 \mathrm{~mm}$, los cuales se pinzaron, cortaron y ligaron con seda 000; arteria espermática izquierda con dilatación aneurismática de $1 \mathrm{~cm}$ y trombosis a lo largo de $10 \mathrm{~cm}$, la cual se resecó (Figs. 1 y 2), sin datos de escroto agudo. Al paciente se le realizó plastia inguinal y resección del aneurisma con ligadura de la arteria testicular, conservando la viabilidad del testículo.

El reporte histopatológico describió aneurisma verdadero de la arteria testicular izquierda con trombo levemente organizado, negativo para malignidad.

Después de la cirugía, el paciente se encuentra en buenas condiciones generales, conservando la viabilidad testicular y sin ninguna complicación aparente.

\section{Discusión}

Existen pocos casos reportados en la literatura de aneurismas verdaderos de la arteria testicular. En el caso aquí reportado, el paciente no tenía ningún antecedente de importancia ni la presencia de fenotipos característicos de síndromes relacionados con el desarrollo de aneurismas, como el síndrome de Marfan, los cuales también pueden desarrollarse en otros procesos mórbidos como las vasculitis, la poliarteritis nodosa y otros procesos vasculares. Sin embargo, no hubo evidencia de inflamación subyacente y no se encontró evidencia de ningún trastorno asociado del tejido conectivo. 


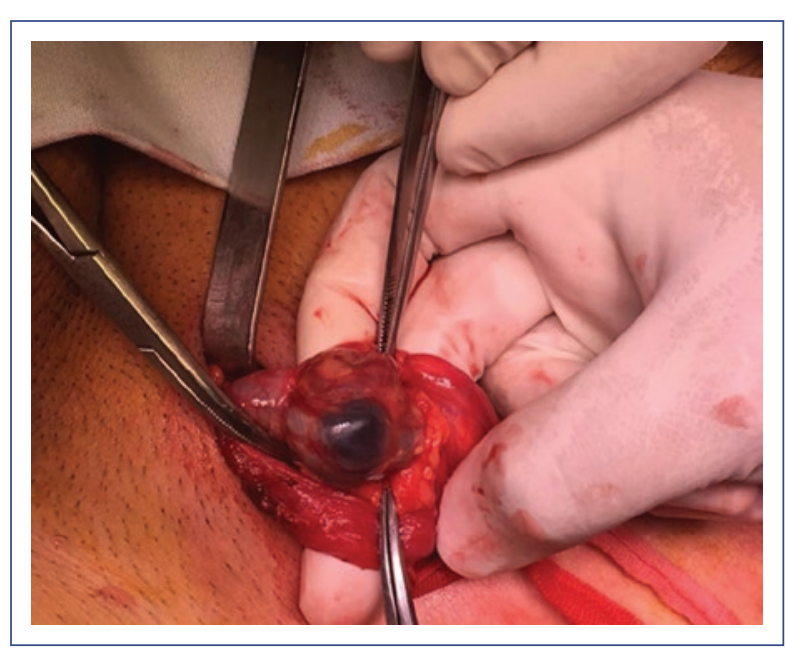

Figura 1. Dilatación de arteria testicular por aneurisma verdadero.

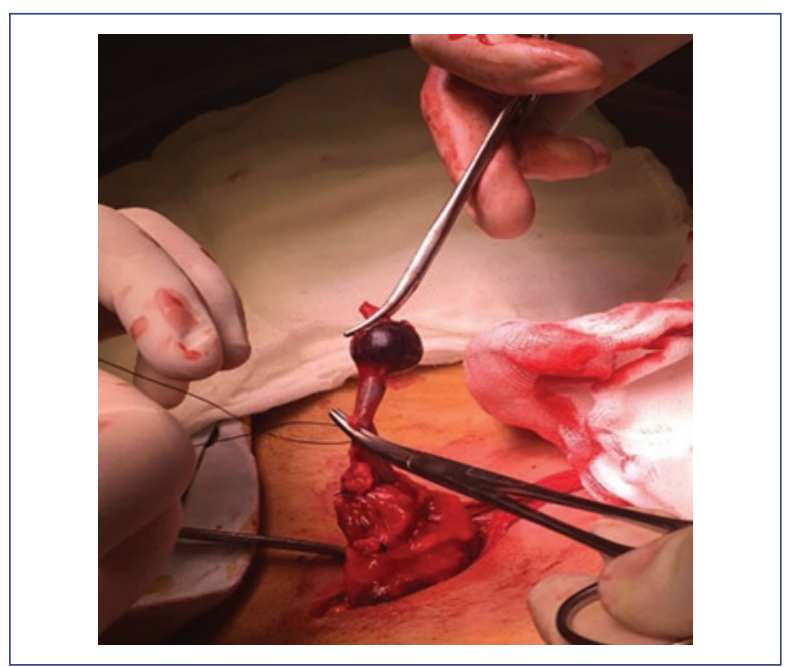

Figura 2. Arteria testicular seccionada.

En un caso reportado en 2014 por Parker y Nangia ${ }^{7}$ en los EE.UU. se describe un paciente con un cuadro de dolor testicular. Por medio de ultrasonido se diagnosticó una lesión sugestiva de pseudoaneurisma de la arteria testicular; se determinó que la etiología del pseudoaneurisma fue una orquitis crónica, lo cual apoya dicha enfermedad como factor de riesgo para su formación. En el presente caso no se logró identificar el aneurisma mediante el ultrasonido, y la etiología se sospechó asociada al traumatismo descrito por el paciente. En el año 2004, Zicherman, et al. ${ }^{8}$ describieron el caso de un paciente de 91 años en quien se diagnosticó un aneurisma de la arteria testicular de manera preoperatoria por medio de ultrasonido; se reportó una masa hipoecoica de $4 \times 2 \times 2.3 \mathrm{~cm}$ en el canal inguinal izquierdo, con un foco anecoico de $7 \mathrm{~mm}$ en su centro y flujo pulsátil por Doppler color compatible con aneurisma. En 1998, en un caso publicado por Reddy, et al. ${ }^{9}$ no se logró identificar la etiología de un aneurisma; se reportó como un hallazgo transoperatorio. No se logró diagnosticar de manera preoperatoria por estudio de imagen ni se determinó la etiología. De los tres casos descritos, este es el más similar al presente reporte, ya que el diagnostico se realizó en el transoperatorio tras la visualización directa de la malformación del vaso sanguíneo.

El paciente de este reporte se presentó a consulta médica por una masa inguinal y dolor esporádico, sin que se lograra documentar la presencia del aneurisma por ultrasonografía. Se decidió realizar plastia inguinal y se encontró un aneurisma verdadero de $1 \mathrm{~cm}$ aproximadamente, el cual se resecó ligando la arteria testicular y conservando la viabilidad del testículo gracias a la circulación colateral. En los estudios de imagen posteriores no se encontraron más hallazgos aneurismáticos.

El diagnóstico preoperatorio suele ser difícil dada la baja prevalencia de la patología y, por lo tanto, la baja sospecha diagnóstica. El diagnóstico diferencial requiere ultrasonografía Doppler, que es hasta el momento el estándar de oro para el diagnóstico.

Debido a la rareza de esta patología y a los pocos casos publicados a escala mundial, no existe un tratamiento bien establecido. En dos de los casos revisados en la literatura se realizó la extirpación quirúrgica; en un tercero, al paciente se le dio seguimiento mediante ecografía, después de la trombosis espontánea del aneurisma.

En el caso que se presenta en este reporte se realizó la resección del aneurisma con ligadura de la arteria testicular, conservando la viabilidad del testículo. Se le dio seguimiento al paciente después de la cirugía por la consulta externa a 1, 3, 6 y 11 meses, realizando ultrasonido testicular para valorar datos de atrofia testicular y descartar datos de necrosis.

\section{Responsabilidades éticas}

Protección de personas y animales. Los autores declaran que para esta investigación no se han realizado experimentos en seres humanos ni en animales.

Confidencialidad de los datos. Los autores declaran que han seguido los protocolos de su centro de trabajo sobre la publicación de datos de pacientes.

Derecho a la privacidad y consentimiento informado. Los autores declaran que han seguido los 
Bol Med Hosp Infant Mex. 2018;75

protocolos de su centro de trabajo sobre la publicación de datos de pacientes.

\section{Conflicto de intereses}

Los autores declaran no tener ningún conflicto de intereses.

\section{Bibliografía}

1. Mujoomdar A, Maheshwari S, Zand F, Sircar K, Mesurolle B. Sonographic diagnosis of a ruptured intratesticular pseudoaneurysm secondary to orchitis. AJR Am J Roentgenol. 2007;189:20-2.

2. Deck KE, Deck A, Waitches GM. Intratesticular pseudoaneurysm after blunt trauma. Rev Am J Roentgenol. 2000;174:1136.
3. Ohmor K, Isokawa Y. Aneurysm of the testicular artery. J Urol. 1994; 151:1646-7.

4. Deaton DH. Arterial aneurysms: general considerations. En: Cronenwett LJ, Johnston KW, editores. Rutherford's vascular surgery. Philadelphia: Elsevier; 2014. p.1990-8.

5. Vásquez DE, Díaz C, Carmona Z, Vásquez FR. Varicocele testicular en adolecentes. Rev Salud Uninorte. 2009;25. Disponible en: http://rcientificas.uninorte.edu.co/index.php/salud/article/viewArticle/217/5785

6. Sakadalakis JE, Colborn GI, Weidman TA, Badalament RA, Scaljon WM Galloway NT, et al. Anatomía quirúrgica de testículo. En: Skandalakis JE, Colborn GL, Weidman TA, Foster RS, Kingsnorth AH, Skandalakis LJ, et al., editores. Skandalakis Cirugía y bases de la anatomía quirúrgica. Madrid: Marbán; 2013. p. 1238-46.

7. Parker WP, Nangia AK. Testicular artery pseudoaneurysm: a case report F1000Res. 2014;3:2

8. Zicherman JM, Mistry KM, Sarokhan CT, S de Carvalho V. CT angiography, sonography, and MRI of aneurysm of the testicular artery. AJR Am J Roentegenol. 2004;182:1088-9.

9. Reddy YP, Murphy JK, Sheridan WG. Spontaneous aneurysm of the testicular artery. Br J Urol. 1998;82:599-600. 